Article

\title{
A Geometric Obstruction for CR-Slant Warped Products in a Nearly Cosymplectic Manifold
}

\author{
Siraj Uddin * and M. Z. Ullah \\ Department of Mathematics, Faculty of Science, King Abdulaziz University, Jeddah 21589, Saudi Arabia; \\ malikzakas@hotmail.com \\ * Correspondence: sshehabaldeen@kau.edu.sa or siraj.ch@gmail.com
}

Received: 11 August 2020; Accepted: 14 September 2020; Published: 19 September 2020

check for updates

\begin{abstract}
In the early 20th century, B.-Y. Chen introduced the concept of CR-warped products and obtained several fundamental results, such as inequality for the length of second fundamental form. In this paper, we obtain B.-Y. Chen's inequality for CR-slant warped products in nearly cosymplectic manifolds, which are the more general classes of manifolds. The equality case of this inequality is also investigated. Furthermore, the inequality is discussed for some important subclasses of CR-slant warped products.
\end{abstract}

Keywords: warped products; CR-slant warped product; nearly cosymplectic manifold

MSC: 53C15; 53C40; 53C42; 53B25

\section{Introduction}

A differentiable manifold $\tilde{M}$ endowed with an almost contact metric structure $(\phi, \xi, \eta, g)$ is said to be nearly cosymplectic if the covariant derivative of the almost contact structure $\phi$ with respect to the Levi-Civita connection $\tilde{\nabla}$ is skew-symmetric, i.e., $\left(\tilde{\nabla}_{X} \phi\right) X=0$, for every vector field $X$ on $\tilde{M}$. These manifolds were defined on the line of nearly Kaehler manifolds and studied by Blair [1], Blair and Showers [2]. In the subsequent literature on this topic, quite important were the papers of $H$. Endo [3,4]. The best known example of a non-cosymplectic nearly cosymplectic manifold is the 5-sphere $S^{5}$ as a totally geodesic hypersurface in $S^{6}$.

Recently, Cappelletti-Montano and Dileo [5] proved that every nearly Sasakian manifold of dimension 5 has an associated nearly cosymplectic structure, thereby showing the close relation between these two notions. Furthermore, They proved that every 5-dimensional nearly cosymplectic manifold is an Einstein manifold with positive scalar curvature. In [6], the authors proved that a non-cosymplectic nearly cosymplectic manifold $\tilde{M}$ of dimension $2 n+1>5$ is locally isometric to one of the Riemannian products: $\mathbb{R} \times \tilde{N}^{2 n}, \tilde{M}^{5} \times \tilde{N}^{2 n-4}$, where $\tilde{N}^{2 n}$ is a non-Kaehler nearly Kaehler manifold, $\tilde{N}^{2 n-4}$ is a nearly Kaehler manifold, and $\tilde{M}^{5}$ is a non-cosymplectic nearly cosymplectic manifold.

On the other hand, in 1969, Bishop and O'Neill introduced the notion of a warped product manifolds to provide a class of complete Riemannian manifolds with negative curvature [7]. This scheme was later applied to semi-Riemannian geometry and the theory of relativity.

Recently, B.-Y. Chen [8] (see also [9]) introduced the concept CR-warped product submanifolds of Kaehler manifolds. He proved that the warped products of the form $M=N^{\perp} \times{ }_{f} N^{T}$ are trivial and the warped products $M=N^{T} \times{ }_{f} N^{\perp}$ exist in Kaehler manifolds, where $N^{T}$ and $N^{\perp}$ are holomorphic and totally real submanifolds of a Kaehler manifold $\tilde{M}$. Motivated by Chen's work on warped products many geometers studied warped product submanifolds of almost Hermitian and contact metric manifolds (for instance, see, [10-15]). In [16], B. Sahin introduced CR-slant warped products named skew CR-warped product submanifolds of Kaehler manifolds and he proved that CR-warped products 
and hemi-slant warped products are particular classes of CR-slant warped products. We refer Chen's books $[17,18]$ for up-to-date survey on warped product manifolds and warped product submanifolds.

In this paper, we study CR-slant warped product submanifolds of nearly cosymplectic manifolds which are the more general classes of contact metric manifolds. We prove that every CR-slant warped product $M=B \times_{f} N^{\theta}$ in a nearly cosymplectic manifold satisfies the following inequality:

$$
\|\sigma\|^{2} \geq \frac{2 s}{9} \cos ^{4} \theta\left\|\nabla^{\perp}(\ln f)\right\|^{2}+4 s\left(\csc ^{2} \theta+\frac{1}{9} \cot ^{2} \theta\right)\left\|\nabla^{T}(\ln f)\right\|^{2},
$$

where $B=N^{T} \times N^{\perp}$, a contact CR-product and $2 s=\operatorname{dim} N^{\theta}$, while $\nabla^{T}(\ln f)$ and $\nabla^{\perp}(\ln f)$ are the gradient components of $\ln f$ along $N^{T}$ and $N^{\perp}$, respectively.

\section{Preliminaries and Basic Results}

An odd dimensional almost contact metric manifold is a smooth manifold $\tilde{M}$ of dimension $2 n+1$, endowed with a structure $(\phi, \xi, \eta, g)$, given by a $(1,1)$ tensor field $\phi$, a vector field $\xi$, a 1-form $\eta$ and a Riemannian metric $g$ satisfying [19]

$$
\phi^{2}=-I+\eta \otimes \xi, \quad \eta(\xi)=1, \quad g(\phi X, \phi Y)=g(X, Y)-\eta(X) \eta(Y)
$$

for all vector fields $X, Y$ on $\tilde{M}$ (see [20] for more details). From the definition it follows that $\phi \xi=0$ and $\eta \circ \phi=0$. Furthermore, $\phi$ is skew-symmetric with respect to $g$, so that the bilinear form $\Phi(X, Y):=g(X, \phi Y)$ defines a 2-form on $\tilde{M}$, called fundamental 2-form. An almost contact metric manifold with $d \eta=2 \Phi$ is called a contact metric manifold. In this case, $\eta$ is a contact form, i.e., $\eta \wedge(d \eta)^{n} \neq 0$ everywhere on $\tilde{M}$.

An almost contact metric manifold $(\tilde{M}, \phi, \xi, \eta, g)$ is called a cosymplectic manifold if $d \eta=0$, $d \Phi=0$ and $N_{\phi}=0$, where $N_{\phi}$ is the Nijenhuis tensor of $\phi$. Equivalently, we have $\tilde{\nabla} \phi=0$. It is known that a cosymplectic manifold is locally a Riemannian product of the real line $\mathbb{R}$ and a Kaehler manifold $\tilde{N}$, which is an integral submanifold of the distribution $\mathcal{D}=\operatorname{Ker}(\eta)$ (see [21] for further details).

A nearly cosymplectic manifold is an almost contact metric manifold $(\tilde{M}, \phi, \xi, \eta, g)$ such that

$$
\left(\tilde{\nabla}_{X} \phi\right) Y+\left(\tilde{\nabla}_{Y} \phi\right) X=0
$$

for all vector fields $X, Y$ on $\tilde{M}$. It is known that in a nearly cosymplectic manifold, the structure vector field $\xi$ is Killing and satisfies $\tilde{\nabla}_{\xi} \xi=0, \tilde{\nabla}_{\xi} \eta=0$ and moreover $g\left(\tilde{\nabla}_{X} \xi, X\right)=0$ for any vector field $X$ tangent to a nearly cosymplectic manifold $\tilde{M}$.

Let $\mathrm{M}$ be a $m$-dimensional manifold isometrically immersed into a Riemannian manifold $\tilde{M}$; denote by the same symbol $g$ the induced metric on $M$. Let $\Gamma(T M)$ be the Lie algebra of vector fields on $M$ and $\Gamma\left(T^{\perp} M\right)$, the set of all vector fields normal to $M$. If we denote by $\nabla$ and $\tilde{\nabla}$, the Levi-Civita connections of $M$ and $\tilde{M}$, respectively, then the Gauss and Weingarten formulas are respectively given by

$$
\tilde{\nabla}_{X} Y=\nabla_{X} Y+\sigma(X, Y), \quad \tilde{\nabla}_{X} N=-A_{N} X+\nabla_{X}^{\perp} N,
$$

for any vector field $X, Y \in \Gamma(T M)$ and $N \in \Gamma\left(T^{\perp} M\right)$, where $\nabla^{\perp}$ is the normal connection in the normal bundle, $\sigma$ is the second fundamental form and $A_{N}$ is the shape operator (corresponding to the normal vector field $N)$ for the immersion of $M$ into $\tilde{M}$. They are related by $g(\sigma(X, Y), N)=g\left(A_{N} X, Y\right)$.

For any $X$ tangent to $M$ and $N$ normal to $M$, we write

$$
\phi X=T X+F X, \quad \phi N=t N+f N
$$


where $T X$ (respectively, $F X$ ) is the tangential (respectively, normal) component of $\phi X$ and $t N$ (respectively, $f N$ ) is the tangential (respectively, normal) component of $\phi N$. Then $T$ is an endomorphism on TM and $F$ is a normal valued 1 -form.

For any $p \in M$ and $\left\{E_{1}, \cdots, E_{m}, \cdots, E_{2 n+1}\right\}$ is an orthonormal frame of $T_{p} \tilde{M}$ such that $E_{1}, \cdots, E_{m}$ are tangent to $M$ at $p$ and $E_{m+1}, \cdots, E_{2 n+1}$ normal to $M$. Then,

$$
\|\sigma\|^{2}=\sum_{i, j=1}^{m} g\left(\sigma\left(E_{i}, E_{j}\right), \sigma\left(E_{i}, E_{j}\right)\right)=\sum_{r=m+1}^{2 n+1} \sum_{i, j=1}^{m} g\left(\sigma\left(E_{i}, E_{j}\right), E_{r}\right)^{2} .
$$

There are two well-known classes of submanifolds, namely $\phi$-invariant submanifolds and $\phi$-anti-invariant submanifolds due to the behaviour of the tangent bundle of a submanifold under the action of the almost contact structure tensor $\phi$ of the ambient manifold. In the case of invariant submanifolds, the tangent space remains invariant under the action of the almost contact structure tensor $\phi$ whereas in case of anti-invariant submanifolds it is mapped into the normal space.

As a generalized class of invariant and anti-invariant submanifolds, B.-Y. Chen introduced slant submanifolds of almost Hermitian manifolds. Later, A. Lotta [22] and Cabrerizo et al. [23] in separate articles extended this study to almost contact metric manifolds.

A submanifold $M$ tangent to the structure vector field $\xi$ is called slant if for every non-zero tangent vector $X$ which is not proportional to $\xi_{p}$, the angle $0 \leq \theta(X) \leq \pi / 2$ between $\phi X$ and $T_{p} M$ is constant (called, slant angle), i.e., $\theta$ is independent of the choice of $X \in \Gamma(T M) \backslash\{\xi, 0\}$ and $p \in M$. If the slant angle is different from 0 and $\pi / 2$, then it is called proper slant.

Another, generalized class of CR-submanifolds and slant submanifolds introduced as semi-slant submanifolds by N. Papaghuic [24]. Later, these submanifolds studied by Cabrerizo et al. [25] in almost contact metric manifolds.

A submanifold $M$ of an almost contact metric manifold $\tilde{M}$ is a semi-slant submanifold if there exist two orthogonal distribution $\mathcal{D}$ and $\mathcal{D}^{\theta}$ on $M$ such that:

(i) $T M$ admits the orthogonal direct decomposition $T M=\mathcal{D} \oplus \mathcal{D}^{\theta} \oplus\langle\xi\rangle$.

(ii) The distribution $\mathcal{D}$ is an invariant distribution, i.e., $\phi(\mathcal{D})=\mathcal{D}$.

(iii) The distribution $\mathcal{D}^{\theta}$ is slant with angle $\theta \neq 0$.

Hemi-slant submanifolds were defined by Carriazo in [26] under the name of anti-slant submanifolds as a particular class of bi-slant submanifolds. A submanifold $M$ of an almost contact metric manifold $\tilde{M}$ is said to be a hemi-slant submanifold if there exists a pair of orthogonal distributions $\mathcal{D}^{\perp}$ and $\mathcal{D}^{\theta}$ on $M$ such that $T M=\mathcal{D}^{\perp} \oplus \mathcal{D}^{\theta} \oplus\left\langle\xi>\right.$ with $\mathcal{D}^{\perp}$ is anti-invariant, i.e., $\phi\left(\mathcal{D}^{\perp}\right) \subset T^{\perp} M$ and $\mathcal{D}^{\theta}$ is a proper slant distribution with angle $\theta$.

Now, we recall the following useful characterization theorem proved in [23].

Theorem 1. Let $M$ be a submanifold of an almost contact metric manifold $\tilde{M}$, such that $\xi \in \Gamma(T M)$. Then, $M$ is slant if and only if there exists a constant $\lambda \in[0,1]$, we have

$$
T^{2}=\lambda(-I+\eta \otimes \xi)
$$

Furthermore, if $\theta$ is the slant angle of $M$, then $\lambda=\cos ^{2} \theta$.

The following relations are easily obtained from Theorem 1:

$$
g(T X, T Y)=\cos ^{2} \theta[g(X, Y)-\eta(X) \eta(Y)]
$$

and

$$
g(F X, F Y)=\sin ^{2} \theta[g(X, Y)-\eta(X) \eta(Y)]
$$


for any $X, Y \in \Gamma(T M)$.

\section{Definitions and Lemmas on CR-Slant Warped Products}

A warped product $B \times_{f} F$ of two Riemannian manifolds $\left(B, g_{B}\right)$ and $\left(F, g_{F}\right)$ is the product manifold $B \times F$ equipped with the warped product metric

$$
g(X, Y)=g_{B}\left(\pi_{1 *} X, \pi_{1 *} Y\right)+\left(f \circ \pi_{1}\right)^{2} g_{F}\left(\pi_{2 *} X, \pi_{2 *} Y\right),
$$

where $f: B \rightarrow(0, \infty)$ is a positive differentiable function on $B$ and $\pi_{1}: M \rightarrow B, \pi_{2}: M \rightarrow F$ are projection maps given by $\pi_{1}(p, q)=p$ and $\pi_{2}(p, q)=q$ for any $(p, q) \in B \times F$ and $*$ denotes the symbol for the tangent map. If function $f$ (called, warping function) is constant, then $M$ is simply a Riemannian product. We know that, for any vector field $X$ on $B$ and a vector field $Z$ on $F$, we have

$$
\nabla_{X} Z=\nabla_{Z} X=X(\ln f) Z,
$$

where $\nabla$ is the Levi-Civita connection on $M$. Notice that on a warped product manifold $M, B$ is totally geodesic and $F$ is totally umbilical in $M$.

In this section, we study CR-slant warped product submanifolds of the form $M=B \times_{f} N^{\theta}$ of a nearly cosymplectic manifold $\tilde{M}$, where $B=N^{T} \times N^{\perp}$, a contact CR-product of invariant and anti-invairant submanifolds of $\tilde{M}$, and $N^{\theta}$ is a slant submanifold. For the simplicity, throughout this paper we denote the corresponding tangent spaces of $N^{T}, N^{\perp}$ and $N^{\theta}$ by $\mathcal{D}, \mathcal{D}^{\perp}$ and $\mathcal{D}^{\theta}$, respectively.

Definition 1. A submanifold $M$ tangent to the structure vector field $\xi$ of an almost contact metric manifold $\tilde{M}$ is called a CR-slant warped product if it is a warped product of the form $M=B \times_{f} N^{\theta}$, where the fiber $N^{\theta}$ is proper slant and the base $B=N^{T} \times N^{\perp}$ is the Riemannian product (called, CR-product) of invariant and anti-invariant submanifolds of $\tilde{M}$.

For a CR-slant warped product $M=B \times_{f} N^{\theta}$ of an almost contact metric manifold $\tilde{M}$, the tangent space is decomposed as:

$$
T M=\mathcal{D} \oplus \mathcal{D}^{\perp} \oplus \mathcal{D}^{\theta} \oplus\langle\xi\rangle
$$

where $\mathcal{D}$ is an invariant distribution, $\mathcal{D}^{\perp}$ is an anti-invariant distribution and $\mathcal{D}^{\theta}$ is a proper slant distribution and $\langle\xi\rangle$ is the 1-dimensional distribution spanned by the structure vector field $\xi$. Clearly, we observe that if $\xi$ along $\mathcal{D}^{\theta}$ then the CR-slant warped product $M=B \times_{f} N^{\theta}$ is trivial as follows: Since $\xi \in \Gamma\left(\mathcal{D}^{\theta}\right)$ is killing on a nearly cosymplectic manifold, from (3) and (10), we find $X(\ln f)=0$ for all $X \in \Gamma(T B)$, i.e., $f$ is constant on $B$.

Furthermore, the normal bundle $T^{\perp} M$ is decomposed as

$$
T^{\perp} M=\phi \mathcal{D}^{\perp} \oplus F \mathcal{D}^{\theta} \oplus \mu,
$$

where $\mu$ is the invariant normal subbundle of $T^{\perp} M$ under $\phi$.

From now, we use the following conventions: $X_{1}, Y_{1} \in \Gamma(\mathcal{D}), \quad X_{2}, Y_{2} \in \Gamma\left(\mathcal{D}^{\perp}\right)$ and $X_{3}, Y_{3} \in \Gamma\left(\mathcal{D}^{\theta}\right)$.

Lemma 1. Let $M=B \times_{f} N^{\theta}$ be a CR-slant warped product submanifold of a nearly cosymplectic manifold $\tilde{M}$ such that $B=N^{T} \times N^{\perp}$ and $\xi \in \Gamma(T B)$. Then, we have

(i) $\xi(\ln f)=0$,

(ii) $g\left(\sigma\left(X_{1}, Y_{1}\right), F X_{3}\right)=0$,

(iii) $g\left(\sigma\left(X_{1}, X_{3}\right), F Y_{3}\right)=\frac{1}{3} X_{1}(\ln f) g\left(T X_{3}, Y_{3}\right)-\phi X_{1}(\ln f) g\left(X_{3}, Y_{3}\right)$,

for any $X_{1}, Y_{1}$ tangent to $N^{T}$ and $X_{3}, Y_{3}$ tangent to $N^{\theta}$. 
Proof. Statements (i) and (ii) were proved in [27] (see Lemma 3.2) but for (iii), by using (1), (3) and (10), we have

$$
g\left(\sigma\left(X_{1}, X_{3}\right), F Y_{3}\right)=g\left(\left(\tilde{\nabla}_{X_{1}} \phi\right) X_{3}, Y_{3}\right)+g\left(\sigma\left(X_{1}, Y_{3}\right), F X_{3}\right) .
$$

Again, from (3), (4) and (10), we obtain

$$
g\left(\sigma\left(X_{1}, X_{3}\right), F Y_{3}\right)=g\left(\left(\tilde{\nabla}_{X_{3}} \phi\right) X_{1}, Y_{3}\right)-\phi X_{1}(\ln f) g\left(X_{3}, Y_{3}\right)-X_{1}(\ln f) g\left(X_{3}, T Y_{3}\right) .
$$

Then, with the help (2), equations (13) and (14) give

$$
2 g\left(\sigma\left(X_{1}, X_{3}\right), F Y_{3}\right)=g\left(\sigma\left(X_{1}, Y_{3}\right), F X_{3}\right)-\phi X_{1}(\ln f) g\left(X_{3}, Y_{3}\right)-X_{1}(\ln f) g\left(X_{3}, T Y_{3}\right) .
$$

By polarization identity, we find

$$
2 g\left(\sigma\left(X_{1}, Y_{3}\right), F X_{3}\right)=g\left(\sigma\left(X_{1}, X_{3}\right), F Y_{3}\right)-\phi X_{1}(\ln f) g\left(X_{3}, Y_{3}\right)+X_{1}(\ln f) g\left(X_{3}, T Y_{3}\right) .
$$

Thus, (iii) follows from the above two last relations.

Following relations are easily obtained by interchanging $X_{1}$ with $\phi X_{1} ; X_{3}$ with $T X_{3}$ and $Y_{3}$ with $T Y_{3}$ with the help of (1) and (6) in Lemma 1 (iii).

$$
\begin{gathered}
g\left(\sigma\left(X_{1}, T X_{3}\right), F Y_{3}\right)=-\frac{1}{3} X_{1}(\ln f) \cos ^{2} \theta g\left(X_{3}, Y_{3}\right)-\phi X_{1}(\ln f) g\left(T X_{3}, Y_{3}\right), \\
g\left(\sigma\left(X_{1}, X_{3}\right), F T Y_{3}\right)=\frac{1}{3} X_{1}(\ln f) \cos ^{2} \theta g\left(X_{3}, Y_{3}\right)-\phi X_{1}(\ln f) g\left(X_{3}, T Y_{3}\right), \\
g\left(\sigma\left(X_{1}, T X_{3}\right), F T Y_{3}\right)=-\frac{1}{3} X_{1}(\ln f) \cos ^{2} \theta g\left(X_{3}, T Y_{3}\right)-\phi X_{1}(\ln f) \cos ^{2} \theta g\left(X_{3}, Y_{3}\right), \\
g\left(\sigma\left(\phi X_{1}, X_{3}\right), F Y_{3}\right)=\frac{1}{3} \phi X_{1}(\ln f) g\left(T X_{3}, Y_{3}\right)+X_{1}(\ln f) g\left(X_{3}, Y_{3}\right), \\
g\left(\sigma\left(\phi X_{1}, T X_{3}\right), F Y_{3}\right)=-\frac{1}{3} \phi X_{1}(\ln f) \cos ^{2} \theta g\left(X_{3}, Y_{3}\right)+X_{1}(\ln f) g\left(T X_{3}, Y_{3}\right), \\
g\left(\sigma\left(\phi X_{1}, X_{3}\right), F T Y_{3}\right)=\frac{1}{3} \phi X_{1}(\ln f) \cos ^{2} \theta g\left(X_{3}, Y_{3}\right)+X_{1}(\ln f) g\left(X_{3}, T Y_{3}\right)
\end{gathered}
$$

and

$$
g\left(\sigma\left(\phi X_{1}, T X_{3}\right), F T Y_{3}\right)=-\frac{1}{3} \phi X_{1}(\ln f) \cos ^{2} \theta g\left(X_{3}, T Y_{3}\right)+X_{1}(\ln f) \cos ^{2} \theta g\left(X_{3}, Y_{3}\right)
$$

Definition 2. A CR-slant warped product $B \times_{f} N^{\theta}$ of an almost contact metric manifold $\tilde{M}$ is called $\mathcal{D} \oplus \mathcal{D}^{\theta}$-mixed totally geodesic if its second fundamental form satisfies $\sigma\left(\mathcal{D}, \mathcal{D}^{\theta}\right)=\{0\}$.

Similarly, $M$ is $\mathcal{D}^{\perp} \oplus \mathcal{D}^{\theta}$-mixed totally geodesic if $\sigma\left(\mathcal{D}^{\perp}, \mathcal{D}^{\theta}\right)=\{0\}$.

Lemma 2. Let $M=B \times_{f} N^{\theta}$ be a CR-slant warped product submanifold of a nearly cosymplectic manifold $\tilde{M}$ such that $B=N^{T} \times N^{\perp}$ and $\xi \in \Gamma(T B)$. Then, we have

(i) $2 g\left(\sigma\left(X_{2}, Y_{2}\right), F X_{3}\right)=g\left(\sigma\left(X_{2}, X_{3}\right), \phi Y_{2}\right)+g\left(\sigma\left(Y_{2}, X_{3}\right), \phi X_{2}\right)$,

(ii) $g\left(\sigma\left(X_{1}, X_{2}\right), F X_{3}\right)=\frac{1}{2} g\left(\sigma\left(X_{1}, X_{3}\right), \phi X_{2}\right)$,

for any $X_{1}$ tangent to $N^{T} ; X_{2}, Y_{2}$ tangent to $N^{\perp}$ and $X_{3}$ tangent to $N^{\theta}$. 
Proof. From (3) and (4), we have

$$
g\left(\sigma\left(X_{2}, Y_{2}\right), F X_{3}\right)=g\left(\tilde{\nabla}_{X_{2}} Y_{2}, F X_{3}\right)=g\left(\left(\tilde{\nabla}_{X_{2}} \phi\right) Y_{2}, X_{3}\right)+g\left(A_{\phi Y_{2}} X_{2}, X_{3}\right)
$$

Interchanging $X_{2}$ and $Y_{2}$, we find

$$
g\left(\sigma\left(X_{2}, Y_{2}\right), F X_{3}\right)=g\left(\left(\tilde{\nabla}_{Y_{2}} \phi\right) X_{2}, X_{3}\right)+g\left(A_{\phi X_{2}} Y_{2}, X_{3}\right)
$$

Then, the first statements follows from (24) and (25) together with (2). For the second part, we have

$$
g\left(\sigma\left(X_{1}, X_{2}\right), F X_{3}\right)=g\left(\tilde{\nabla}_{X_{1}} X_{2}, F X_{3}\right)=g\left(\left(\tilde{\nabla}_{X_{1}} \phi\right) X_{2}, X_{3}\right)+g\left(A_{\phi X_{2}} X_{1}, X_{3}\right)-X_{1}(\ln f) g\left(X_{2}, T X_{3}\right) .
$$

By orthogonality of distributions, we find

$$
g\left(\sigma\left(X_{1}, X_{2}\right), F X_{3}\right)=g\left(\left(\tilde{\nabla}_{X_{1}} \phi\right) X_{2}, X_{3}\right)+g\left(\sigma\left(X_{1}, X_{3}\right), \phi X_{2}\right) .
$$

On the other hand, we also have

$g\left(\sigma\left(X_{1}, X_{2}\right), F X_{3}\right)=g\left(\tilde{\nabla}_{X_{2}} X_{1}, F X_{3}\right)=g\left(\left(\tilde{\nabla}_{X_{2}} \phi\right) X_{1}, X_{3}\right)-\phi X_{1}(\ln f) g\left(X_{2}, X_{3}\right)-X_{1}(\ln f) g\left(X_{2}, T X_{3}\right)$.

Again, by orthogonality of the distributions, we get

$$
g\left(\sigma\left(X_{1}, X_{2}\right), F X_{3}\right)=g\left(\left(\tilde{\nabla}_{X_{2}} \phi\right) X_{1}, X_{3}\right)
$$

Hence, from (26), (27) and (2), we get the desired result.

Lemma 3. For a $C R$-slant warped product $M=B \times_{f} N^{\theta}$ in a nearly cosymplectic manifold $\tilde{M}$, we have

$$
g\left(\sigma\left(X_{3}, Y_{3}\right), \phi X_{2}\right)=\frac{1}{3} X_{2}(\ln f) g\left(X_{3}, T Y_{3}\right)+g\left(\sigma\left(X_{2}, X_{3}\right), F Y_{3}\right)
$$

for any $X_{2}$ tangent to $N^{\perp}$ and $X_{3}, Y_{3}$ tangent to $N^{\theta}$.

Proof. For any $X_{2} \in \Gamma\left(\mathcal{D}^{\perp}\right)$ and $X_{3}, Y_{3} \in \Gamma\left(\mathcal{D}^{\theta}\right)$, by using (3) and (10), we find

$$
g\left(\sigma\left(X_{2}, X_{3}\right), F Y_{3}\right)=g\left(\left(\tilde{\nabla}_{X_{3}} \phi\right) X_{2}, Y_{3}\right)+g\left(A_{\phi X_{2}} X_{3}, Y_{3}\right)-X_{2}(\ln f) g\left(X_{3}, T Y_{3}\right)
$$

On the other hand, we also have by using (3) and (10) and orthogonality of vector fields

$$
g\left(\sigma\left(X_{2}, X_{3}\right), F Y_{3}\right)=g\left(\left(\tilde{\nabla}_{X_{2}} \phi\right) X_{3}, Y_{3}\right)+g\left(A_{F X_{3}} X_{2}, Y_{3}\right)
$$

From (29) and (30) with (2), we derive

$$
2 g\left(\sigma\left(X_{2}, X_{3}\right), F Y_{3}\right)=g\left(\sigma\left(X_{3}, Y_{3}\right), \phi X_{2}\right)+g\left(\sigma\left(X_{2}, Y_{3}\right), F X_{3}\right)-X_{2}(\ln f) g\left(X_{3}, T Y_{3}\right)
$$

By polarization identity, we obtain

$$
2 g\left(\sigma\left(X_{2}, Y_{3}\right), F X_{3}\right)=g\left(\sigma\left(X_{3}, Y_{3}\right), \phi X_{2}\right)+g\left(\sigma\left(X_{2}, X_{3}\right), F Y_{3}\right)+X_{2}(\ln f) g\left(X_{3}, T Y_{3}\right)
$$

Then, the required result follows from (31) and (32).

By interchanging $X_{3}$ with $T X_{3}$ and $Y_{3}$ with $T Y_{3}$, one can get the following relations.

$$
g\left(\sigma\left(T X_{3}, Y_{3}\right), \phi X_{2}\right)=\frac{1}{3} X_{2}(\ln f) \cos ^{2} \theta g\left(X_{3}, Y_{3}\right)+g\left(\sigma\left(X_{2}, T X_{3}\right), F Y_{3}\right)
$$




$$
\begin{gathered}
g\left(\sigma\left(X_{3}, T Y_{3}\right), \phi X_{2}\right)=-\frac{1}{3} X_{2}(\ln f) \cos ^{2} \theta g\left(X_{3}, Y_{3}\right)+g\left(\sigma\left(X_{2}, X_{3}\right), F T Y_{3}\right) \\
g\left(\sigma\left(T X_{3}, T Y_{3}\right), \phi X_{2}\right)=\frac{1}{3} X_{2}(\ln f) \cos ^{2} \theta g\left(X_{3}, T Y_{3}\right)+g\left(\sigma\left(X_{2}, T X_{3}\right), F T Y_{3}\right) .
\end{gathered}
$$

\section{Main Results}

In this section, we present our main results of the paper. First, we have the following non-existence theorem of proper CR-slant warped products.

Theorem 2. Let $M=B \times_{f} N^{\theta}$ be a CR-slant warped product in a nearly cosymplectic manifold $\tilde{M}$ such that $B=N^{T} \times N^{\perp}$. If $M$ is $\mathcal{D} \oplus \mathcal{D}^{\theta}$-mixed totally geodesic then, $f$ depends only on $N_{\perp}$, i.e., $f$ is constant along $N_{T}$.

Proof. For a $\mathcal{D} \oplus \mathcal{D}^{\theta}$-mixed totally geodesic CR-slant warped product, from Lemma 1 (iii) and (21), we derive

$$
\left(\cos ^{2} \theta-9\right) \phi X_{1}(\ln f) g\left(X_{3}, Y_{3}\right)=0,
$$

for any $X_{1} \in \Gamma(\mathcal{D})$ and $X_{3}, Y_{3} \in \Gamma\left(\mathcal{D}^{\theta}\right)$. Since $g$ is a Riemannian metric, then we find either $\cos \theta= \pm 3$ which is impossible, or $\phi X_{1}(\ln f)=0$, i.e., $f$ is constant along $N^{T}$, which proves the theorem completely.

Now, we establish a sharp estimation for the length of the second fundamental form by using the following frame field for a CR-slant warped product.

Let $M=B \times_{f} N^{\theta}$ be a $m$-dimensional CR-slant warped product submanifold of a $2 n+1$ dimensional nearly cosymplectic manifold $\tilde{M}$ such that $B$ is the Riemannian product of an invariant submanifold $N^{T}$ and an anti-invariant submanifold $N^{\perp}$ in $\tilde{M}$. Let the corresponding tangent space of $N^{T}, N^{\perp}$ and $N^{\theta}$ respectively are $\mathcal{D}, \mathcal{D}^{\perp}$ and $\mathcal{D}^{\theta}$. If $\operatorname{dim} \mathcal{D}=2 \alpha+1, \operatorname{dim} \mathcal{D}^{\perp}=\gamma$ and $\operatorname{dim} \mathcal{D}^{\theta}=2 \beta$, then the tangent bundle TM is spanned by the following orthonormal frame fields $\mathcal{D}=\operatorname{Span}\left\{E_{1}, \cdots, E_{\alpha}, E_{\alpha+1}=\phi E_{1}, \cdots, E_{2 \alpha}=\phi E_{\alpha}, E_{2 \alpha+1}=\xi\right\}$, $\mathcal{D}^{\perp}=\operatorname{Span}\left\{E_{2 \alpha+2}=\hat{E}_{1}, \cdots, E_{2 \alpha+\gamma+1}=\hat{E}_{\gamma}\right\}$ and $\mathcal{D}^{\theta}=\operatorname{Span}\left\{E_{2 \alpha+\gamma+2}=E_{1}^{*}, \cdots, E_{2 \alpha+\gamma+\beta+1}=\right.$ $\left.E_{\beta}^{*}, E_{2 \alpha+\gamma+\beta+2}=E_{\beta+1}^{*}=\sec \theta T E_{1}^{*}, \cdots, E_{m}=E_{2 \beta}^{*}=\sec \theta T E_{\beta}^{*}\right\}$. Furthermore, the normal subbundles of $T^{\perp} M$ are spanned by $\phi \mathcal{D}^{\perp}=\operatorname{Span}\left\{E_{m+1}=\tilde{E}_{1}=\phi \hat{E}_{1}, \cdots, E_{m+\gamma}=\tilde{E}_{\gamma}=\phi \hat{E}_{\gamma}\right\}$, $F \mathcal{D}^{\theta}=\operatorname{Span}\left\{E_{m+\gamma+1}=\tilde{E}_{\gamma+1}=\csc \theta F E_{1}^{*}, \cdots, E_{m+\gamma+\beta}=\tilde{E}_{\gamma+\beta}=\csc \theta F E_{\beta^{\prime}}^{*} E_{m+\gamma+\beta+1}=\right.$ $\left.\tilde{E}_{\gamma+\beta+1}=\csc \theta \sec \theta F T E_{1}^{*}, \cdots, E_{m+\gamma+2 \beta}=\tilde{E}_{\gamma+2 \beta}=\csc \theta \sec \theta F T E_{\gamma}^{*}\right\}$ and $\mu=\operatorname{Span}\left\{E_{m+\gamma+2 \beta+1}=\right.$ $\left.\tilde{E}_{\gamma+2 \beta+1}, \cdots, E_{2 n+1}=\tilde{E}_{2 n+1-m-\gamma-2 \beta}\right\}$.

Theorem 3. Let $M=B \times_{f} N^{\theta}$ be a $\mathcal{D}^{\perp} \oplus \mathcal{D}^{\theta}$-mixed totally geodesic $C R$-slant warped product submanifold of a nearly cosymplectic manifold $\tilde{M}$ such that $B=N^{T} \times N^{\perp}$. Then, the second fundamental form $\sigma$ of $M$ satisfies:

$$
\|\sigma\|^{2} \geq \frac{2 \beta}{9} \cos ^{4} \theta\left\|\nabla^{\perp}(\ln f)\right\|^{2}+4 \beta\left(\csc ^{2} \theta+\frac{1}{9} \cot ^{2} \theta\right)\left\|\nabla^{T}(\ln f)\right\|^{2},
$$

where $\beta=\frac{1}{2} \operatorname{dim} N^{\theta}$ and $\nabla^{\perp}(\ln f)$ and $\nabla^{T}(\ln f)$ are the gradient components of $\ln f$ along $N^{\perp}$ and $N^{T}$, respectively.

Moreover, if the equality sign holds in (37), then $N^{T}$ and $N^{\perp}$ are totally geodesic submanifolds of $\tilde{M}$ and $N^{\theta}$ is a totally umbilical submanifold of $\tilde{M}$. Furthermore, $M$ is also a $\mathcal{D} \oplus \mathcal{D}^{\perp}$-mixed totally geodesic submanifold of $\tilde{M}$ but never be a $\mathcal{D} \oplus \mathcal{D}^{\theta}$-mixed totally geodesic and hence $M$ is not minimal in $\tilde{M}$. 
Proof. From (5), we have

$$
\begin{aligned}
\|\sigma\|^{2} & =\sum_{r=m+1}^{2 n+1} \sum_{i, j=1}^{m} g\left(\sigma\left(E_{i}, E_{j}\right), E_{r}\right)^{2} \\
& =\sum_{r=m+1}^{m+\gamma} \sum_{i, j=1}^{m} g\left(\sigma\left(E_{i}, E_{j}\right), E_{r}\right)^{2}+\sum_{r=m+\gamma+1}^{m+\gamma+2 \beta} \sum_{i, j=1}^{m} g\left(\sigma\left(E_{i}, E_{j}\right), E_{r}\right)^{2} \\
& +\sum_{r=m+\gamma+2 \beta+1}^{2 n+1} \sum_{i, j=1}^{m} g\left(\sigma\left(E_{i}, E_{j}\right), E_{r}\right)^{2} .
\end{aligned}
$$

Leaving the third term and decompose first two terms in the right hand side of (39) for the considered orthonormal frame fields, we derive

$$
\begin{aligned}
\|\sigma\|^{2} & \geq \sum_{r=1}^{\gamma} \sum_{i, j=1}^{2 \alpha+1} g\left(\sigma\left(E_{i}, E_{j}\right), \phi \hat{E}_{r}\right)^{2}+\sum_{r=1}^{\gamma} \sum_{i, j=1}^{\gamma} g\left(\sigma\left(\hat{E}_{i}, \hat{E}_{j}\right), \phi \hat{E}_{r}\right)^{2} \\
& +\sum_{r=1}^{\gamma} \sum_{i, j=1}^{2 \beta} g\left(\sigma\left(E_{i}^{*}, E_{j}^{*}\right), \phi \hat{E}_{r}\right)^{2}+2 \sum_{r=1}^{\gamma} \sum_{i=1}^{2 \alpha+1} \sum_{j=1}^{\gamma} g\left(\sigma\left(E_{i}, \hat{E}_{j}\right), \phi \hat{E}_{r}\right)^{2} \\
& +2 \sum_{r=1}^{\gamma} \sum_{i=1}^{2 \alpha+1} \sum_{j=1}^{2 \beta} g\left(\sigma\left(E_{i}, E_{j}^{*}\right), \phi \hat{E}_{r}\right)^{2}+2 \sum_{r=1}^{\gamma} \sum_{i=1}^{\gamma} \sum_{j=1}^{2 \beta} g\left(\sigma\left(\hat{E}_{i}, E_{j}^{*}\right), \phi \hat{E}_{r}\right)^{2} \\
& +\sum_{r=\gamma+1}^{\gamma+2 \beta} \sum_{i, j=1}^{2 \alpha+1} g\left(\sigma\left(E_{i}, E_{j}\right), \tilde{E}_{r}\right)^{2}+\sum_{r=\gamma+1}^{\gamma+2 \beta} \sum_{i, j=1}^{q} g\left(\sigma\left(\hat{E}_{i}, \hat{E}_{j}\right), \tilde{E}_{r}\right)^{2} \\
& +\sum_{r=\gamma+1}^{\gamma+2 \beta} \sum_{i, j=1}^{2 \beta} g\left(\sigma\left(E_{i}^{*}, E_{j}^{*}\right), \tilde{E}_{r}\right)^{2}+2 \sum_{r=\gamma+1}^{\gamma+2 \beta} \sum_{i=1}^{2 \alpha+1} \sum_{j=1}^{\gamma} g\left(\sigma\left(E_{i}, \hat{E}_{j}\right), \tilde{E}_{r}\right)^{2} \\
& +2 \sum_{r=\gamma+1}^{\gamma+2 \beta} \sum_{i=1}^{2 \alpha+1} \sum_{j=1}^{2 \beta} g\left(\sigma\left(E_{i}, E_{j}^{*}\right), \tilde{E}_{r}\right)^{2}+2 \sum_{r=\gamma+1}^{\gamma+2 \beta} \sum_{i=1}^{\gamma} \sum_{j=1}^{2 \beta} g\left(\sigma\left(\hat{E}_{i}, E_{j}^{*}\right), \tilde{E}_{r}\right)^{2} .
\end{aligned}
$$

Using Lemma 1, relations (17)-(23), Lemmas 2 and 3 with the relations (33)-(35), after computations, we derive

$$
\begin{aligned}
\|\sigma\|^{2} & \geq \frac{2 \beta}{9} \cos ^{4} \theta \sum_{r=1}^{\gamma}\left(\hat{E}_{r}(\ln f)\right)^{2}+10 \sum_{r=\gamma+1}^{\gamma+2 \beta} \sum_{i=1}^{2 \alpha+1} \sum_{j=1}^{\gamma} g\left(\sigma\left(E_{i}, \hat{E}_{j}\right), \tilde{E}_{r}\right)^{2} \\
& +4 \beta \csc ^{2} \theta \sum_{i=1}^{\alpha}\left[\left(E_{i}(\ln f)\right)^{2}+\left(\phi E_{i}(\ln f)\right)^{2}\right]+\frac{4 \beta}{9} \cot ^{2} \theta \sum_{i=1}^{\alpha}\left[\left(E_{i}(\ln f)\right)^{2}+\left(\phi E_{i}(\ln f)\right)^{2}\right] .
\end{aligned}
$$

Since $\sum_{i=1}^{\alpha}\left(\phi E_{i}(\ln f)\right)^{2}=\sum_{i=1}^{\alpha} g\left(\nabla \ln f, \phi E_{i}\right)^{2}=\sum_{i=\alpha+1}^{2 \alpha} g\left(\nabla \ln f, E_{\alpha+i}\right)^{2}$. Using this fact in (40), we obtain

$$
\begin{aligned}
\|\sigma\|^{2} & \geq \frac{2 \beta}{9} \cos ^{4} \theta\left\|\nabla^{\perp}(\ln f)\right\|^{2}+4 \beta\left(\csc ^{2} \theta+\frac{1}{9} \cot ^{2} \theta\right) \sum_{i=1}^{2 \alpha+1}\left(E_{i}(\ln f)\right)^{2} \\
& -4 \beta\left(\csc ^{2} \theta+\frac{1}{9} \cot ^{2} \theta\right) \xi(\ln f) .
\end{aligned}
$$

The required inequality follows from (41) by using the fact $\xi(\ln f)=0$. For the equality, from the leaving third term in r.h.s. of (37), we find $\sigma(X, Y)$ has no components in $\mu$ for all $X, Y$ tangent to $M$. Furthermore, from the leaving first term and vanishing seventh term in r.h.s. of (39) with the above fact that $\sigma$ has no components in $\mu$, we find

$$
\sigma(\mathcal{D}, \mathcal{D})=\{0\}
$$


Also, from the leaving fourth term in r.h.s. of (39) and the second term in r.h.s. of (40) with the fact that $\sigma$ has no components in $\mu$, we find

$$
\sigma\left(\mathcal{D}, \mathcal{D}^{\perp}\right)=\{0\}
$$

From the hypothesis of the theorem

$$
\sigma\left(\mathcal{D}^{\perp}, \mathcal{D}^{\theta}\right)=\{0\}
$$

And from the leaving second term and vanishing eighth term in r.h.s. of (39) with this fact that $\sigma$ has no components in $\mu$, we conclude that

$$
\sigma\left(\mathcal{D}^{\perp}, \mathcal{D}^{\perp}\right)=\{0\}
$$

Furthermore, from the leaving ninth term in r.h.s. of (39) with the fact that $\sigma$ has no components in $\mu$, we obtain

$$
\sigma\left(\mathcal{D}^{\theta}, \mathcal{D}^{\theta}\right) \subset \phi \mathcal{D}^{\perp}
$$

With the help of above facts and the fact that $B$ is totally geodesic and $N^{\theta}$ is totally umbilical in $M[7,8]$, we conclude that $N^{T}$ and $N^{\perp}$ are totally geodesic submanifolds of $\tilde{M}$, while $N^{\theta}$ is a totally umbilical submanifold of $\tilde{M}$. Furthermore, from (43) $M$ is also $\mathcal{D} \oplus \mathcal{D}^{\perp}$-mixed totally geodesic. Moreover, from Theorem 2, $M$ can never be a $\mathcal{D} \oplus \mathcal{D}^{\theta}$-mixed totally geodesic. Hence, the theorem is proved completely.

As applications of Theorem 3, we have the following results.

If $\operatorname{dim} N^{T}=0$ in Theorem 3, then we have

Theorem 4. Let $M=N^{\perp} \times_{f} N^{\theta}$ be a mixed totally geodesic proper hemi-slant warped product of a nearly cosymplectic manifold $\tilde{M}$. Then, the second fundamental form $\sigma$ of $M$ satisfies

$$
\|\sigma\|^{2} \geq \frac{2 s}{9} \cos ^{4} \theta\left\|\nabla^{\perp}(\ln f)\right\|^{2},
$$

where $2 s=\operatorname{dim} N^{\theta}$ and $\nabla^{\perp}(\ln f)$ is the gradient of $\ln f$.

Moreover, if the equality holds in (47), then $N^{\perp}$ is a totally geodesic submanifold of $\tilde{M}$ and $N^{\theta}$ is a totally umbilical submanifold of $\tilde{M}$. Furthermore, $M$ is minimal in $\tilde{M}$.

If $N^{\perp}=\{0\}$ in Theorem 3 , then we state the following theorem.

Theorem 5. Let $M=N^{T} \times_{f} N^{\theta}$ be a warped product semi-slant submanifold of a nearly cosymplectic manifold $\tilde{M}$. Then, the second fundamental form $\sigma$ of $M$ satisfies

$$
\|\sigma\|^{2} \geq 4 s\left(\csc ^{2} \theta+\frac{1}{9} \cot ^{2} \theta\right)\left\|\nabla^{T}(\ln f)\right\|^{2}
$$

where $s=\frac{1}{2} \operatorname{dim} N^{\theta}$ and $\nabla^{T}(\ln f)$ is the gradient of $\ln f$.

Moreover, if the equality holds in (48), then $N^{T}$ is a totally geodesic submanifold of $\tilde{M}$ and $N^{\theta}$ is a totally umbilical submanifold of $\tilde{M}$. Furthermore, $M$ is never a mixed totally geodesic submanifold and hence $M$ is not minimal in $\tilde{M}$.

Notice that Theorem 5 was proved in [27] which is a special case of Theorem 3. Also, in the above statement we improve the equality case of the main theorem of [27].

Theorem 5 implies the following theorem proved in [28]. 
Theorem 6. Let $M=N^{T} \times_{f} N^{\perp}$ be a contact CR-warped product submanifold of a nearly cosymplectic manifold $\tilde{M}$. Then, the second fundamental form $\sigma$ of $M$ satisfies

$$
\|\sigma\|^{2} \geq 2 s\left\|\nabla^{T}(\ln f)\right\|^{2},
$$

where $s=\operatorname{dim} N^{\perp}$ and $\nabla^{T}(\ln f)$ is the gradient of $\ln f$.

Moreover, if the equality holds in (49), then $N^{T}$ is a totally geodesic submanifold of $\tilde{M}$ and $N^{\perp}$ is a totally umbilical submanifold of $\tilde{M}$. Furthermore, $M$ is a minimal submanifold of $\tilde{M}$.

\section{Conclusions}

In $[27,28]$, we studied contact CR-warped product and semi-slant warped product submanifolds of nearly cosymplectic manifolds and obtained B.-Y. Chen's inequalities. As a generalised class of these submanifolds, in this paper, we study CR-slant warped products in nearly cosymplectic manifolds and establish a geometric inequality (Theorem 3) which generalizes Theorem 6 for contact CR-warped products, Theorem 5 for semi-slant warped products and Theorem 4 for hemi-slant warped products.

Author Contributions: The main contents of the manuscript are contributed by the first author and the second author helped in tidy calculations and the typing of this manuscript. All authors have read and agreed to the published version of the manuscript.

Funding: Research and development office (RDO) at the ministry of Education, Kingdom of Saudi Arabia. Grant no (HIQI-50-2019).

Acknowledgments: This Project was funded by the research and development office (RDO) at the ministry of Education, Kingdom of Saudi Arabia, under Grant no (HIQI-50-2019). The authors also, acknowledge with thanks research and development office (RDO-KAU) at King Abdulaziz University for technical support.

Conflicts of Interest: The authors declare no conflict of interest.

\section{References}

1. Blair, D.E. Almost contact manifolds with Killing structure tensors. Pac. J. Math. 1971, 39, 285-292. [CrossRef]

2. Blair, D.E.; Showers, D.K. Almost contact manifolds with Killing structure tensors. II. J. Differ. Geom. 1974, 9,577-582. [CrossRef]

3. Endo, H. On the curvature tensor of nearly cosymplectic manifolds of constant $\phi$-sectional curvature. An. Stiit. Univ. Al. I. Cuza Iasi. Mat. (NS) 2005, 51, 439-454.

4. Endo, H. On the first Betti number of certain compact nearly cosymplectic manifolds. J. Geom. 2012, 103, $231-236$. [CrossRef]

5. Cappelletti-Montano, B.; Dileo, G. Nearly Sasakian geometry and SU(2)-structures. Ann. Mat. Pura Appl. 2016, 195, 897-922. [CrossRef]

6. De Nicola, A.; Dileo, G.; Yudin, I. On nearly Sasakian and nearly cosymplectic manifolds. Annali di Matematica Pura ed Applicata 2018, 197, 127-138. [CrossRef]

7. Bishop, R.L.; O'Neill, B. Manifolds of negative curvature. Trans. Am. Math. Soc. 1969, 145, 1-49. [CrossRef]

8. Chen, B.-Y. Geometry of warped product CR-submanifolds in Kaehler manifold. Monatsh. Math. 2001, 133, 177-195. [CrossRef]

9. Chen, B.-Y. Geometry of warped product CR-submanifolds in Kaehler manifolds II. Monatsh. Math. 2001, 134, 103-119. [CrossRef]

10. Chen, B.-Y.; Uddin, S. Warped product pointwise bi-slant submanifolds of Kaehler manifolds. Publ. Math. Debrecen 2018, 92, 183-199. [CrossRef]

11. Chen, B.-Y.; Uddin, S.; Al-Solamy, F.R. Geometry of pointwise CR-slant warped products in Kaehler manifolds. Rev. Union Mat. Argentina 2020, 61.

12. Hasegawa, I.; Mihai, I. Contact CR-warped product submanifolds in Sasakian manifolds. Geom. Dedicata 2003, 102, 143-150. [CrossRef]

13. Naghi, M.F.; Mihai, I.; Uddin, S.; Al-Solamy, F.R. Warped product skew CR-submanifolds of Kenmotsu manifolds and their applications. Filomat 2018, 32, 1-24. [CrossRef] 
14. Uddin, S.; Kon, S.H.; Khan, M.A.; Singh, K. Warped product semi-invariant submanifolds of nearly cosymplectic manifolds. Math. Probl. Eng. 2011, 230374. [CrossRef]

15. Uddin, S.; Chen, B.-Y.; Al-Solamy, F.R. Warped product bi-slant immersions in Kaehler manifolds. Mediterr. J. Math. 2017, 14, 95. [CrossRef]

16. Sahin, B. Skew CR-warped products of Kaehler manifolds. Math. Commun. 2010, 15, 188-204.

17. Chen, B.-Y. Pseudo-Riemannian Geometry, S-Invariants and Applications; World Scientific: Hackensack, NJ, USA, 2011.

18. Chen, B.-Y. Differential Geometry of Warped Product Manifolds and Submanifolds; World Scientific: Hackensack, NJ, USA, 2017.

19. Chinea, D.; Gonzalez, C. A classification of almost contact metric manifolds. Ann. Mat. Pura Appl. 1990, 156, 15-36. [CrossRef]

20. Blair, D.E. Riemannian geometry of contact and symplectic manifolds. In Progress in Mathematics, 2nd ed.; Birkhauser: Boston, MA, USA, 2010; p. 203.

21. Cappelletti-Montano, B.; De Nicola, A.; Yudin, I. A survey on cosymplectic geometry. Rev. Math. Phys. 2013, 25, 1343002. [CrossRef]

22. Lotta, A. Slant submanifolds in contact geometry Bull. Math. Soc. Rouman. 1996, 39, 183-198.

23. Cabrerizo, J.L.; Carriazo, A.; Fernandez, L.M.; Fernandez, M. Slant submanifolds in Sasakian manifolds. Glasgow Math. J. 2000, 42, 125-138. [CrossRef]

24. Papaghiuc, N. Semi-slant submanifolds of Kaehlerian manifold. Ann. St. Univ. Iasi 1994, 9, 55-61.

25. Cabrerizo, J.L.; Carriazo, A.; Fernandez, L.M.; Fernandez, M. Semi-slant submanifolds of a Sasakian manifold. Geom. Dedicata 1999, 78, 183-199. [CrossRef]

26. Carriazo, A. New Developments in Slant Submanifolds Theory; Narosa Publishing House: New Delhi, Indian, 2002.

27. Uddin, S.; Mustafa, A.; Wong, B.R.; Ozel, C. A geometric inequality for warped product semi-slant submanifolds of nearly cosymplectic manifolds. Rev. Un. Mat. Argentina 2014, 55, 55-69.

28. Uddin, S.; Khan, K.A. An nequality for contact CR-warped product submanifolds of nearly cosymplectic manifolds. J. Inequal. Appl. 2012 2012, 304. [CrossRef]

(C) 2020 by the authors. Licensee MDPI, Basel, Switzerland. This article is an open access article distributed under the terms and conditions of the Creative Commons Attribution (CC BY) license (http:/ / creativecommons.org/licenses/by/4.0/). 